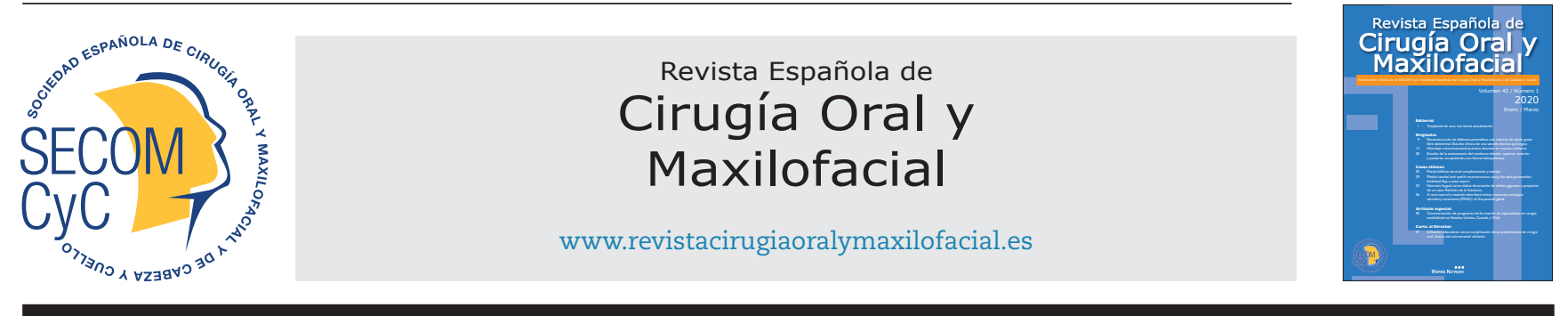

\title{
Original
}

\section{Abordaje transconjuntival: primera elección en trauma orbitario}

\section{Christian Pedemonte Trewhela $a^{a^{*}}$, Andrea Carmona Avendaño ${ }^{a}$ Edgardo González Mora ${ }^{a}$, Ilich Vargas Farren ${ }^{a}$, Claudio Huentequeo ${ }^{b}$ y Alfredo Noguera ${ }^{b}$}

${ }^{a}$ Servicio de Cirugía y Traumatología Maxilofacial, Hospital Clínico Mutual de Seguridad. Santiago, Región Metropolitana, Chile. ${ }^{b}$ Cirugía y Traumatología Maxilofacial, Universidad de los Andes, Santiago, Región Metropolitana, Chile

\section{INFORMACIÓN DEL ARTÍCULO}

Historia del artículo:

Recibido el 16 de febrero de 2019

Aceptado el 22 de abril de 2019

\section{Palabras clave:}

Abordaje transconjuntival, complicaciones, fracturas orbitarias, órbita.

\section{R E S U M E N}

Objetivo: Describir la eficacia del abordaje transconjuntival para el tratamiento de fracturas orbitarias con compromiso del suelo de la órbita y las posibles complicaciones asociadas a este abordaje.

Material y método: Es un estudio retrospectivo descriptivo en base a 98 pacientes con fractura de órbita donde se utilizó el abordaje transconjuntival, en el Servicio de Cirugía Maxilofacial del Hospital Clínico Mutual de Seguridad de Santiago de Chile, entre enero del 2012 y abril del 2017. Se determinó la tasa de éxito de dicha técnica y las variables con mayor predisposición a producir complicaciones. Los criterios de inclusión fueron pacientes con fracturas del complejo maxilofacial con compromiso de una o más paredes orbitarias. Se utilizó el test de Fisher para el análisis de datos, siendo significativo un $\mathrm{p}$ value $<0,05$.

Resultados: Los resultados obtenidos con el abordaje transconjuntival utilizado para el tratamiento de fracturas del complejo orbitomalar demuestran una alta tasa de éxito (94\%) en relación con las escasas complicaciones (6\%). Usando el test Fisher, las complicaciones estuvieron asociadas a fracturas maxilofaciales complejas por accidentes de alta energía, que traen como consecuencia gran destrucción del complejo orbitario, afectándose frecuentemente el aparato lagrimal, en reintervenciones usando el mismo acceso del abordaje, y en variaciones de la técnica en que se utilizó el abordaje transconjuntival con cantotomía lateral y/o retrocaruncular.

Conclusiones: El abordaje transconjuntival es una excelente alternativa en fracturas del complejo orbitomaxilo malar, especialmente para acceder al suelo de la órbita y al reborde infraorbitario, por sus mínimas complicaciones, pronóstico favorable y técnica de baja complejidad.

\footnotetext{
*Autor para correspondencia:

Correo electrónico: capedemonte@gmail.com (Christian Pedemonte).
} 
Transconjuntival approach: first choice in orbit trauma

\section{A B S T R A C T}

Keywords:

Transconjunctival approach, complications, orbital fractures, orbit.
Objective: To describe the efficacy of the transconjunctival approach in orbital and orbital floor fractures and the possible complications associated with this approach.

Material and method: This is a retrospective descriptive study based on 98 patients with orbital fractures where the transconjunctival approach was used in the Maxillofacial Surgery Service of Mutual de Seguridad Hospital, Santiago - Chile, between January 2012 and April 2017. The success rate of this technique and the variables with greater predisposition to generate complications were determined. The inclusion criteria were patients with fractures of the maxillofacial complex with involvement of one or more orbital walls. Fisher's test was used for the data analysis, $\mathrm{p}$ - value $<0.05$ being significant.

Results: Results obtained in our study demonstrate a high success rate (94\%) in relation to the transconjunctival approach used for orbitozygomatic complex fractures treatments, with few complications observed (6\%). Using the Fisher's test, complications were associated with complex maxillofacial fractures due to high energy accidents, which resulted in great destruction of the orbital complex, frequently affecting the lacrimal apparatus, in reinterventions using the same approach access and in variations of the technique in which the transconjunctival approach with lateral cantotomy and / or retrocaruncular extension was used.

Conclusions: The transconjunctival approach is an excellent alternative in orbitozygomatic maxilar complex fractures, especially to access the orbital floor and the infraorbital rim, due to its minimal complications and favorable prognosis.

\section{INTRODUCCIÓN}

El acceso para el tratamiento de las fracturas orbitomalares debe permitir buena visibilidad de las fracturas y una correcta reducción tridimensional de estas. Para acceder a fracturas orbitarias se han descrito múltiples abordajes, tales como el abordaje coronal, palpebral superior, abordajes transcutáneos inferiores tradicionales como el subciliar, subtarsal y palpebral inferior, y el abordaje transconjuntival que puede ser preseptal o retroseptal, pudiendo extenderse a la pared lateral a través de una cantotomía lateral y a la pared medial por medio de un abordaje retrocaruncular o transcaruncular ${ }^{1-3}$.

El abordaje transconjuntival fue descrito por primera vez por Bourguet y cols. ${ }^{4}$ en 1924, en una blefaroplastia cosmética, para extirpar tejido graso. Actualmente es utilizado para el acceso al esqueleto orbitomalar, ganando amplia aceptación en el tratamiento de fracturas orbitarias por su moderada complejidad quirúrgica y buen resultado estético y funcional postquirúrgico ${ }^{2,5-8}$. Según la literatura, dentro de las ventajas de este abordaje encontramos: 1) cicatriz imperceptible; 2) mínima malposición cantal; 3) igual o mayor exposición del suelo orbitario que abordajes transcutáneos; 4) permite extender el campo operatorio hacia la pared medial y hacia la pared lateral, y 5) menor tiempo quirúrgico ${ }^{3,6,9}$.

Las complicaciones más frecuentes se deben a incisiones incorrectas, reacción de cuerpo extraño atribuidas al cierre del periostio y/o conjuntiva palpebral y reintervenciones. Estas complicaciones se pueden manifestar como: epífora, entropion, ectropion, retracción del párpado inferior, edema persistente, quemosis, irritación de la carúncula, laceración canalicular o del saco lagrimal, fibrosis por formación de bridas cicatrizales y/o granuloma conjuntival ${ }^{3,5-12}$.
El objetivo principal de este estudio es describir la eficacia del abordaje transconjuntival para el tratamiento de fracturas orbitarias con compromiso del suelo de la órbita y describir las posibles complicaciones oculares asociadas a este abordaje.

\section{PACIENTES Y MÉTODOS}

Este es un estudio descriptivo retrospectivo en base a 98 pacientes con diagnóstico previo de fractura orbitaria, a los cuales se les realizó un abordaje transconjuntival retroseptal para el acceso a estas. Las cirugías fueron realizadas por el equipo de Cirugía Maxilofacial del Hospital Clínico Mutual de Seguridad de Santiago de Chile, entre los meses de noviembre de 2012 y abril de 2017. Se evaluaron complicaciones postoperatorias con un periodo de seguimiento de 6 meses postintervención quirúrgica.

Se analizaron las tomografías computadas maxilofaciales sin contraste preoperatorias (cortes axiales, coronales y sagitales) de todos los pacientes de este estudio, permitiendo recopilar información sobre el tipo de fractura presente. Posteriormente se revisaron los protocolos operatorios excluyendo aquellos pacientes en los que no se realizó abordaje transconjuntival y se registraron las complicaciones en relación con el abordaje acorde a las evoluciones postoperatorias descritas en la ficha clínica de los participantes.

Los criterios de inclusión fueron: pacientes mayores de 18 años, presencia de fracturas del complejo orbitomalar u orbitarias, fracturas uni- o bilaterales con compromiso del suelo orbitario, pudiendo estar extendido a la pared medial y/o lateral con un periodo de seguimiento mínimo de 6 meses. Se consi- 
deraron como criterios de exclusión pacientes que presentaron fracturas del techo y/o pared lateral de la órbita aisladas.

Para determinar la necesidad de corrección quirúrgica de la órbita se consideró: 1) clínicamente diplopía persistente y/o enoftalmos mayor de $2 \mathrm{~mm}$, disestesia infraorbitaria, escalón palpable del reborde infraorbitario, restricción de los movimientos del globo ocular; 2) evidencia en la tomografía computada maxilofacial sin contraste de herniación de tejido blando y/o atrapamiento del recto medial y/o recto inferior o defecto orbitario extenso mayor $2 \mathrm{~cm}$.

Se realizó un seguimiento postoperatorio a los 7 días, 1, 3 y 6 meses y, en algunos casos, más de 6 meses. Las complicaciones presentadas dentro de la primera semana fueron consideradas inmediatas, entre los 7 a 30 días mediatas y las complicaciones tardías fueron posterior a 30 días postintervención quirúrgica.

Todos los pacientes seleccionados para este estudio firmaron un consentimiento informado. Este estudio fue aprobado por el Comité de Ética del Hospital Clínico Mutual de Seguridad que está de acuerdo con la declaración de Helsinki.

Las variables analizadas fueron: 1) edad; 2) género; 3) causa del accidente; 4) sitio de fractura; 5) tipo de abordaje quirúrgico: transconjuntival, transconjuntival con cantotomía lateral, transconjuntival retrocaruncular con cantotomía lateral y transconjuntival retrocaruncular; 6) corrección volumen orbitario: instalación de malla preformada, prótesis personalizada de titanio o prótesis personalizada de polieteretercetona (PEEK); 7) presencia de sutura en la conjuntiva; 8) reintervenciones, y 9) complicaciones relacionadas con el abordaje.

Se calificaron como reintervenciones las cirugías realizadas en una segunda etapa para corregir volumen orbitario y diplopía. Cabe destacar que todos los pacientes fueron evaluados pre y postoperatoriamente por un oftalmólogo del Hospital Clínico Mutual de Seguridad.

\section{Técnica quirúrgica}

Todos los participantes del estudio fueron intervenidos quirúrgicamente por el mismo equipo de Cirugía Maxilofacial del Hospital Clínico Mutual de Seguridad en pabellón central bajo anestesia general. Previo a la intervención, se realizó la prueba ocular de ducción forzada para evidenciar atrapamiento muscular. Se realizó un abordaje transconjuntival retroseptal con técnica directa en todos los casos, pudiendo existir adicionalmente dos variantes según la necesidad de ampliar el campo operatorio, los cuales son: 1) cantotomía lateral, que favorece el acceso a la pared lateral orbitaria y permite fijar mallas de reconstrucción o prótesis personalizada; este acceso podría combinarse con una cantolisis de ser necesario para favorecer la relajación del párpado inferior o para trasponer el canto lateral; 2) acceso retrocaruncular, que permite libre acceso a la pared medial y posterior reconstrucción con malla preformada o una prótesis fabricada a medida.

Para visualizar el campo operatorio, se utilizan separadores de desmarres para retraer el párpado inferior, cuidando de no dañar el sistema de drenaje lagrimal. El globo ocular es protegido con un protector corneal o con un retractor ocular maleable. Previo a realizar el abordaje, se infiltra a nivel del fórnix inferior 1,8 ml de solución de lidocaína al 2 \% con epinefrina en una relación de 1:100.000. Si se acompaña de cantotomía lateral o retrocaruncular se infiltra anestésico local tanto en la conjuntiva lateral como medial.

Utilizando un retractor maleable se identifica el reborde infraorbitario para realizar el abordaje transconjuntival retroseptal. A continuación, se retraen el tarso y el orbicular con retractores de desmarres sobre el reborde infraorbitario y con un bisturí frío hoja $\mathrm{n} .^{\circ} 15$ se realiza una única incisión en la conjuntiva a nivel del fórnix inferior, desde la carúncula medial al fórnix lateral, atravesando la conjuntiva, hasta llegar al periostio del reborde infraorbitario siempre manteniendo contacto óseo. Luego se eleva el periostio exponiendo el reborde orbitario inferior y en dirección hacia el suelo de la órbita (Figura 1). El retractor maleable protege la grasa orbital, por lo que la necesidad de disección a través de la grasa orbitaria es mínima, sin riesgos virtualmente de sangrado o lesión de los músculos extraoculares. En caso de extenderse con un acceso retrocaruncular, se debe buscar la cresta lacrimal posterior llevándolo a una posición más medial, retrayendo con una valva maleable suavemente la carúncula y el aparato lagrimal. Para evitar escindir el músculo, el cual provocaría una alteración de la motilidad ocular generalmente reversible, se debe realizar una incisión en S itálica en la conjuntiva, lo más cercano al reborde infraorbitario o identificarlo y evitar su corte o desinserción, pese a esto, comúnmente es seccionado justo por debajo de la carúncula durante el acceso.

Se identifica fácilmente el rasgo de fractura, teniendo acceso al suelo y reborde orbitario, además de la pared medial al utilizar su variante retrocaruncular y/o pared lateral al realizar una cantotomía lateral (Figura 2). Se realiza reducción de las fracturas y se instalan placas de osteosíntesis a nivel del reborde infraorbitario y, en pacientes con fracturas de suelo de órbita o pared medial, se reconstruye con mallas de titanio (Figura 3) y, en casos más severos y secuelas postraumatismo, se opta por prótesis orbitarias personalizadas.

Al finalizar la reconstrucción orbitaria, en todos los pacientes se realizó la prueba de ducción forzada para comprobar la movilidad sin atrapamiento. Finalmente, preferiblemente el abordaje transconjuntival no es suturado. En cambio, en algunos pacientes siempre se sutura con cantotomía lateral; se realizó una sutura suspensoria del periostio y la conjuntiva ocular.

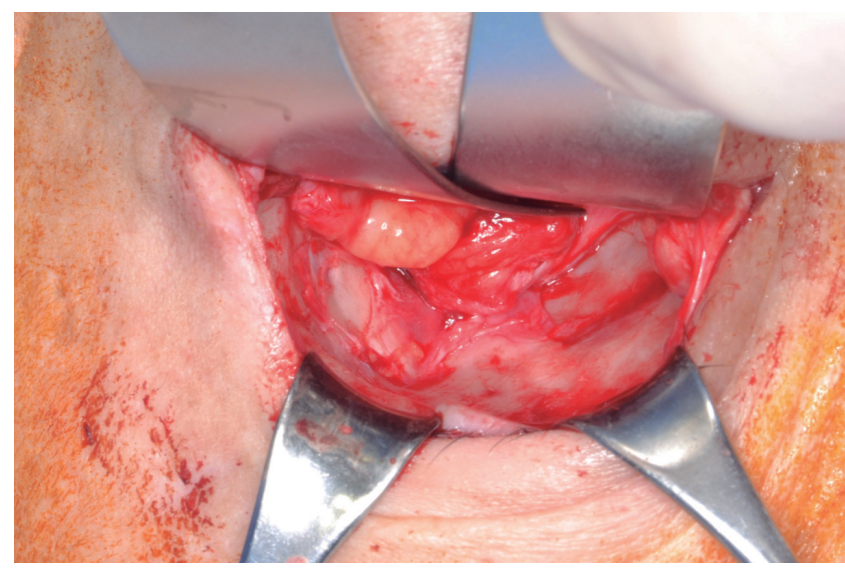

Figura 1. Exposición de sitio quirúrgico. Amplia exposición del suelo de órbita tras realizar el abordaje transconjuntival. 


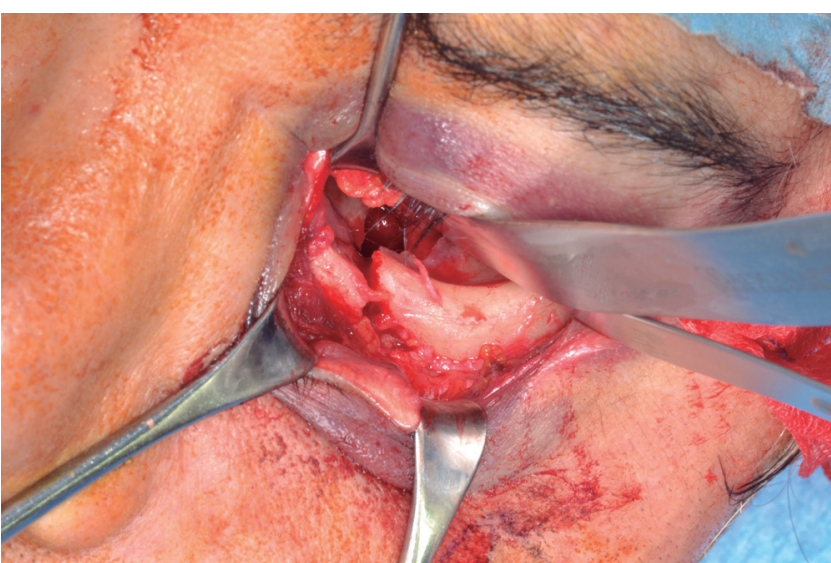

Figura 2. Identificación de rasgo de fractura. Se identifica el rasgo de fractura fácilmente a nivel del reborde infraorbitario y pared medial.

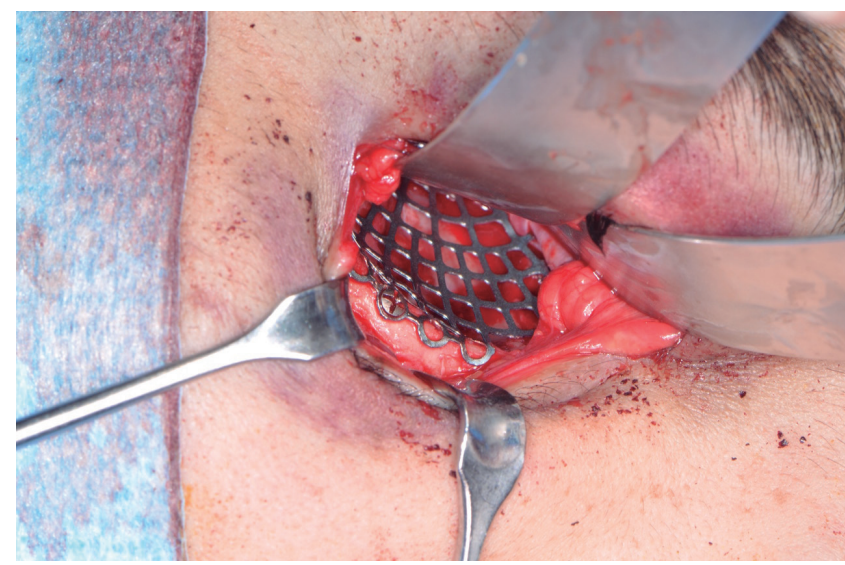

Figura 3. Reconstrucción orbitaria. Paciente con fractura de suelo de órbita, donde la visibilidad y exposición que brinda el abordaje permite adaptar la malla de titanio cubriendo el defecto.

En todos los pacientes se utilizaron cintas adhesivas de papel como apósito compresivo.

\section{Cuidados postoperatorios}

Es importante considerar que la pupila puede aparecer midriática en el postoperatorio inmediato por la anestesia local infiltrada. Se prescribe de forma postoperatoria antibioterapia, analgésicos, antinflamatorios y corticoides.

Es importante instaurar un protocolo de ejercicios oculares de forma temprana y el chequeo postoperatorio inmediato de la agudeza visual.

\section{Análisis estadístico}

Los datos obtenidos fueron registrados en una tabla Excel especificando las variables a correlacionar. Se definió si el abordaje transconjuntival se realizó de manera aislada o asociada a una cantotomía lateral, variación retrocaruncular o ambos y, además, se determinó la presencia de complicaciones inmediatas, mediatas y tardías referidas en la ficha clínica de cada participante.

Se estableció si existe alguna correlación entre el abordaje transconjuntival y las diferentes variables analizadas para presentar complicaciones, utilizando el análisis estadístico Test de Fisher en el programa STATA (StataCorp, College Station, $\mathrm{TX})$, donde un $\mathrm{p}$ value $<0,05$ fue considerado significativo.

\section{RESULTADOS}

Entre noviembre de 2012 hasta abril de 2017 se intervinieron quirúrgicamente a 284 pacientes con un total de 415 intervenciones realizadas en el territorio maxilofacial. De estas, 298 intervenciones fueron realizadas a nivel del tercio superior y/o medio facial. En 168 pacientes hubo compromiso de, al menos, una de las paredes orbitarias. Finalmente, 98 pacientes $(n=98)$ fueron abordados mediante un acceso transconjuntival retroseptal correspondientes a 136 intervenciones.

El rango etáreo de la muestra del estudio fue entre 21 y 81 años, con una edad media de 45 años, en una relación 9:1 entre sexo masculino y femenino, respectivamente. La causa más frecuente de este tipo de fracturas se asoció con accidentes de alta energía (56\%).

Los diagnósticos más frecuentes en pacientes donde se utilizó el abordaje transconjuntival son fracturas orbitomaxilo malar (52\%), seguido por fracturas orbitarias aisladas $(20 \%)$, fracturas fronto orbitomaxilo malar (10\%) y otras (18\%).

Para abordar las fracturas orbitarias en relación con el suelo de la órbita, pared medial y pared lateral, se utilizó el abordaje transconjuntival con diferentes variaciones, siendo el más frecuente el acceso transconjuntival con cantotomía lateral (69\%), seguido por el abordaje transconjuntival retrocaruncular con cantotomía lateral (17\%), el abordaje transconjuntival sin extensión lateral o retrocaruncular (13\%) y, por último, el transconjuntival retrocaruncular (2\%).

En las intervenciones quirúrgicas para reconstruir la morfología orbitaria, se instalaron con mayor frecuencia mallas preformadas (85 \%). En una segunda instancia, con el fin de tratar secuelas producto del traumatismo se instalaron prótesis fabricadas a medida de titanio (11\%) y de polieteretercetona (PEEK) (4\%).

De acuerdo con el protocolo quirúrgico, en la mayoría de las intervenciones no se realizó sutura de la conjuntiva en los abordajes transconjuntivales retroseptales (79\%).

Del total de la muestra estudiada $(n=98)$ correspondiente a 136 intervenciones, el abordaje transconjuntival retroseptal presentó una alta tasa de éxito clínico (94\%), brindando una excelente exposición al reborde infraorbitario y suelo de la órbita. La incisión de cantotomía lateral permitió explorar el reborde infraorbitario y la pared lateral de la órbita hasta la región frontocigomática. El abordaje retrocaruncular facilitó la visualización del campo operatorio hasta la pared medial de la órbita en aquellos pacientes que presentaron compromiso de esta.

Las complicaciones que se encontraron asociadas a las diferentes variaciones de la técnica del abordaje transconjuntival retroseptal fueron: 1) epífora, 2) ectropion, 3) granuloma conjuntival, 4) fístula y 5) entropion (Figura 4). 


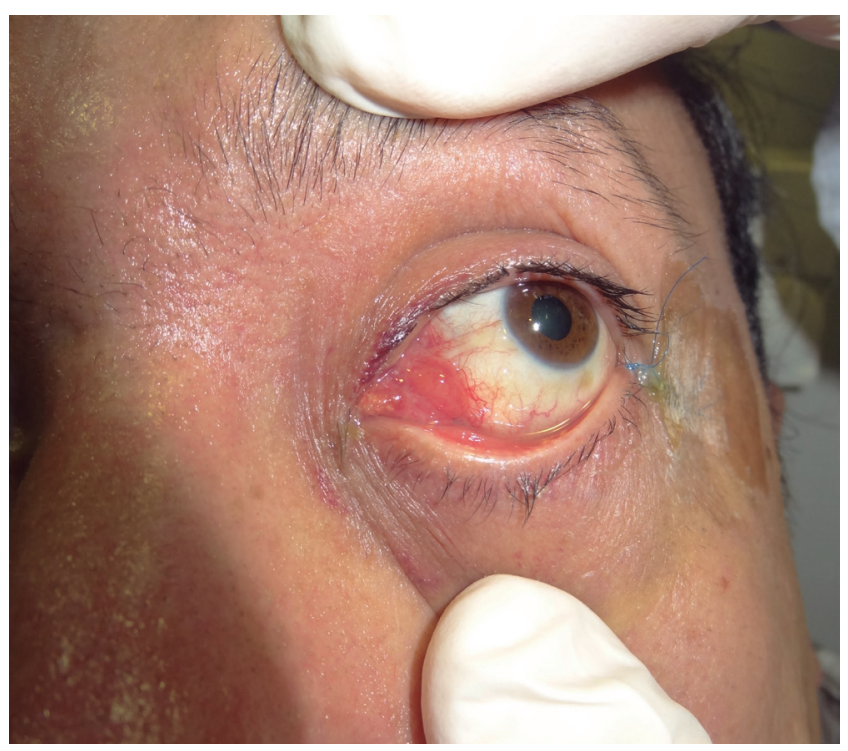

Figura 4. Granuloma conjuntival. Se observa granuloma conjuntival asociado a sutura suspensoria.

En relación con las complicaciones presentadas en la muestra estudiada, 6 pacientes (6\%) presentaron complicaciones postoperatorias tardías relacionadas al abordaje (Tabla I). De estos 6 pacientes, 3 presentaron complicaciones de menos de 6 meses de evolución las cuales fueron autorresolutivas y 3 de ellos presentaron complicaciones de más de 1 año de evolución. Estas últimas requirieron intervención quirúrgica para su resolución.

Según el test de Fisher, en cuanto a la edad, género, tipo de fractura y sutura del abordaje no se encontraron diferencias estadísticamente significativas en relación con las complicaciones por abordaje transconjuntival ( $p>0,05)$. Por otro lado, una vez que se analizaron las complicaciones se encontró mayor morbilidad en asociación con accidentes de alta energía, mayor número de cirugías orbitarias por pacientes (reintervenciones), donde el abordaje transconjuntival estuvo asociado a cantotomía lateral y/o extensión retrocaruncular y a la instalación de elementos de reconstrucción orbitaria $(\mathrm{p}<0,05)$

\section{DISCUSIÓN}

En este estudio se evaluaron 98 pacientes a los cuales se les realizó un abordaje transconjuntival retroseptal para exponer el suelo y reborde infraorbitario y así reconstruir la órbita fracturada. Los resultados obtenidos muestran una alta tasa de éxito (94\%) en relación con las complicaciones asociadas a este abordaje (6\%). Cabe destacar que el entropion consignado en uno de los pacientes después de haber utilizado el abordaje transconjuntival puede estar influenciado por una laceración a nivel del párpado inferior producto del trauma. Todas las complicaciones referidas en este estudio lograron resolverse de manera espontánea o mediante cirugía.

El abordaje transconjuntival retroseptal presentó variaciones según cada caso (cantotomía lateral y/o extensión retro- caruncular) brindando un amplio acceso a las fracturas en la totalidad de la muestra estudiada. La incisión en la conjuntiva palpebral debe ser realizada mediante una técnica quirúrgica atraumática para no dañar el tarso o septo y producir una distorsión postoperatoria del párpado inferior o al globo ocular que aumenta el riesgo de una lesión del músculo oblicuo inferior ${ }^{3,13,14}$.

Las causas de retracción del párpado inferior después de una intervención de suelo de la órbita son multifactoriales, donde tanto la intervención quirúrgica como el mismo trauma pueden contribuir a la contractura del septo orbitario y desplazar el párpado inferior hacia abajo o ectropion, que puede estar influenciado por: manejo inadecuado de la piel y tejidos, insuficiencia lamelar anterior, hematoma, edema de párpado, adherencias del septo orbitario, contractura de cicatriz, conexión cicatricial entre el tabique orbitario, el músculo orbicular y el tejido circundante, laxitud horizontal del margen del párpado, debilitamiento del músculo pretarsal, disección amplia del periostio anterior y la pérdida del tono muscular ${ }^{5,13}$. Si se produce la retracción postoperatoria del párpado inferior, debe manejarse de forma conservadora mediante ejercicios de cierre forzado de párpados y masaje agresivo, donde la gran mayoría de estos casos logra resolverse ${ }^{8,13}$.

El riesgo de desarrollar retracción del párpado postoperatorio también varía con el tipo de abordaje utilizado para exponer el reborde orbitario inferior y el suelo de la órbita ${ }^{3,5}$. Lo que se vio reflejado en nuestros resultados en relación con el tipo de abordaje utilizado, donde hubo una mayor incidencia de complicaciones en variaciones de la técnica del abordaje transconjuntival, es decir, cuando se acompañaba de una extensión retrocaruncular y/o cantotomía lateral. Ningún paciente presentó complicaciones en relación con el abordaje transconjuntival tradicional, por lo que, un factor importante a considerar es la laxitud del párpado, que se ve afectado por la cantotomía lateral y la retracción de los tejidos para permitir una correcta visualización, asimismo la variante retrocaruncular se asocia a la dificultad de evitar el saco lagrimal y respetar estructuras anatómicas como el músculo de Horner y el músculo oblicuo inferior ${ }^{3,6,15,16}$.

La expansión del abordaje transconjuntival mediante procedimientos quirúrgicos, como la cantotomía lateral o la cantólisis, pueden ocasionar un cambio en la inclinación del canto, mayor riesgo de mal posicionamiento y asimetría del párpado inferior e incluso entropion ${ }^{3,8,13}$.

Por otro lado, de acuerdo con nuestro protocolo quirúrgico, no se realiza la sutura del abordaje, con excepción de aquellos casos donde se realizó una tracción excesiva intraoperatoria o se combinó con una extensión retrocaruncular y, ocasionalmente, en pacientes reintervenidos. Observamos que en 3 de los 4 pacientes en los que se realizó sutura de la conjuntiva, se presentaron complicaciones asociadas al abordaje; sin embargo, solo se pudo atribuir directamente a un paciente, ya que todos estos procedimientos se realizaron en pacientes con fracturas extensas y/o conminutas producto de traumas de alta energía, los cuales requirieron abordajes más extensos, donde además la mayoría de los procedimientos fueron reintervenciones.

El cierre sin suturas de la conjuntiva palpebral parece ser una alternativa efectiva, ya que reduce el tiempo operatorio y disminuye incidencia de complicaciones, ya que descomprimi- 


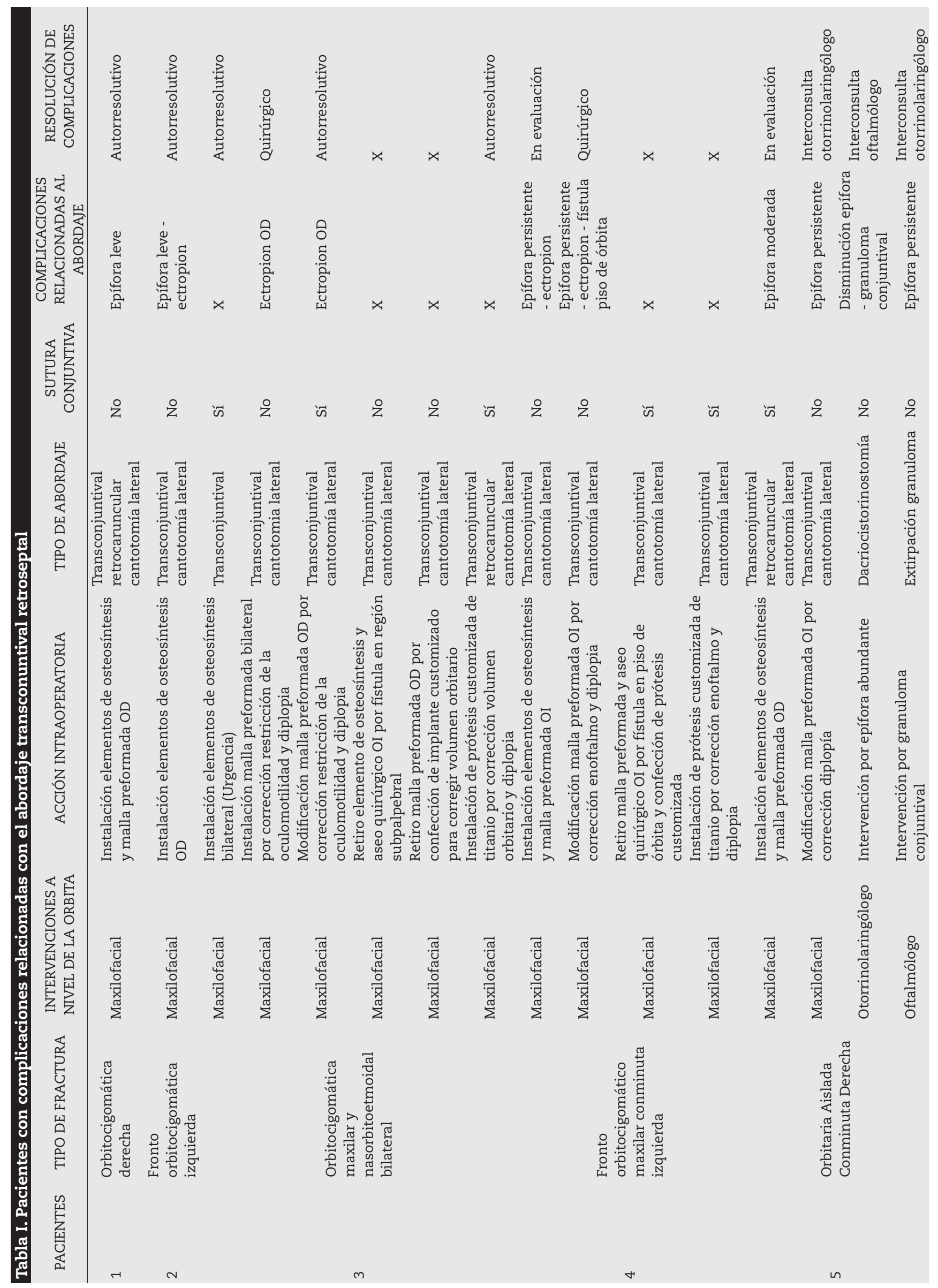




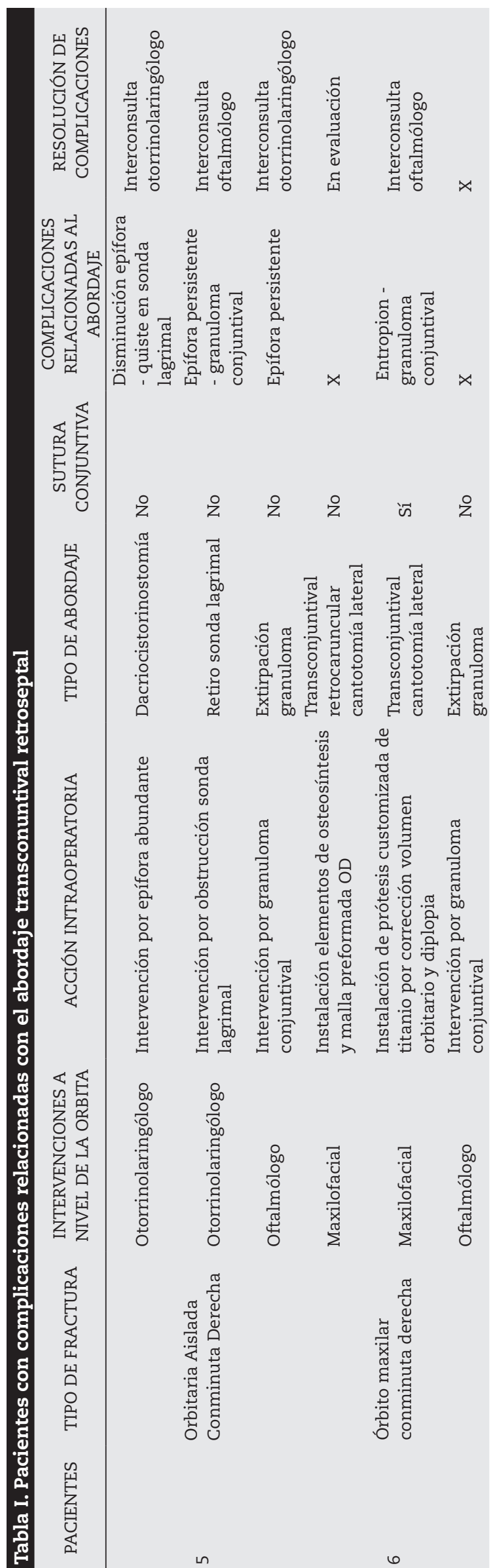

ría la órbita favoreciendo el drenaje del edema y/o hemorragia postoperatoria asociada ${ }^{5,6}$.

Nuestro estudio también reveló que gran parte de las complicaciones observadas se presentaron en pacientes reintervenidos, usando el abordaje transconjuntival retroseptal en el mismo sitio quirúrgico, donde repetidas incisiones a nivel de la conjuntiva pueden ser un factor de riesgo para desarrollar complicaciones postoperatorias. Sin embargo, recomendamos la técnica retroseptal porque requiere menor tiempo operatorio, se puede realizar fácilmente, proporciona un excelente acceso y buenos resultados estéticos, con baja incidencia de complicaciones.

\section{CONCLUSIONES}

Las complicaciones postoperatorias observadas estuvieron asociadas a trauma de alta energía, extensión retrocaruncular y/o cantotomía lateral y pacientes reintervenidos. Recomendamos este abordaje como primera elección en fracturas del complejo orbitomaxilo malar, especialmente para acceder al suelo de la órbita y reborde infraorbitario, por sus mínimas complicaciones, pronóstico favorable y posibilidad de extenderse hacia lateral y medial en base a cantotomía lateral y extensión retrocaruncular, respectivamente.

\section{AGRADECIMIENTOS}

Agradecemos al Dr. Benjamín Martínez por su participación y ayuda con las estadísticas en nuestro estudio.

\section{CONFLICTO DE INTERESES}

Ni los autores ni ningún miembro de su familia inmediata tienen una relación financiera o de interés (actualmente o en los últimos 12 meses) con cualquier entidad de producción, comercialización, reventa o distribución de productos para el cuidado de la salud o servicios consumidos por, o utilizados en, los pacientes.

\section{FINANCIACIÓN}

No hemos recibido financiación para realizar este estudio.

\section{BIBLIOGRAFÍA}

1. Rajkumar K, Mukhopadhyay P, Sinha R, Bandyopadhyay TK. 'Y' Modification of the Transconjunctival Approach for Management of Zygomatic Complex Fractures: A Prospective Analysis. J Maxillofac Oral Surg. 2016;15(1):45-51. DOI: 10.1007/s12663-015-0781-6.

2. Giraddi G, Syed M. Preseptal transconjunctival vs. subciliary approach in treatment of infraorbital rim and floor fractures. Ann Maxillofac Surg. 2012;2(2):136-40. DOI: 10.4103/2231-0746.101338.

3. Martou G, Antonyshyn OM. Advances in surgical approaches to the upper facial skeleton. Curr Opin Otolaryngol Head Neck Surg. 2011;19(4):242-7. DOI: 10.1097/MOO.0b013e3283 47 f895.

4. Bourguet J. Les hernies graisseuse de l'orbite: notre traitement chirurgical (Surgical treatment of fatty hernias of the orbit). Bull Acad Nat Med. 1924;92:1227-32. 
5. Vaibhav N, Keerthi R, Nanjappa M, Ashwin DP, Reyazulla MA, Gopinath AL, et al. Comparison of 'sutureless' Transconjunctival and Subciliary Approach for Treatment of Infraorbital Rim Fractures: a Clinical Study. J Maxillofac Oral Surg. 2016;15(3):35562. DOI: 10.1007/s12663-015-0835-9.

6. Vaibhav N, Abhishek G, Nanjappa M, Keerthi R. "Sutureless" transconjunctival approach for infraorbital rim fractures. Contemp Clin Dent. 2015; 6(Suppl 1):S56-S58. DOI: 10.4103/0976237X.152939

7. Uemura T, Watanabe H, Masumoto K, Chuman T, Satake Y, Yanai $\mathrm{T}$, et al. Transconjunctival approach for zygomatic fracture: A single surgeon's experience of more than 20 years. Plast Reconstr Surg Glob Open. 2016;4(6):e757. DOI: 10.1097/ GOX.0000000000000748.

8. Ridgway EB, Chen C, Lee BT. Acquired entropion associated with the transconjunctival incision for facial fracture management. J Craniofac Surg. 2009;20(5):1412-5. DOI: 10.1097/ SCS.0b013e3181aee3ee.

9. Santosh BS, Giraddi G. Transconjunctival preseptal approach for orbital floor and infraorbital rim fracture. J Maxillofac Oral Surg. 2001;10(4):301-5. DOI: 10.1007/s12663-011-0246-5.

10. Kumar S, Shubhalaksmi S. Clinical outcome following use of transconjunctival approach in reducing orbitozygomaticomaxillary complex fractures. Contemp Clin Dent. 2016;7(2):1639. DOI: 10.4103/0976-237X.183067.
11. Pausch NC, Sirintawat N, Wagner R, Halama D, Dhanuthai K. Lower eyelid complications associated with transconjunctival versus subciliary approaches to orbital floor fractures. Oral Maxillofac Surg. 2016;20(1):51-5. DOI: 10.1007/s10006-015-0526-1.

12. Olate S, Palmieri C, Moraes M. Chemosis as complication in transconjunctival approach for orbital trauma. J Korean Assoc Oral Maxillofac Surg. 2017;43(1):42-5. DOI: 10.5125/jkaoms.2017.43.1.42.

13. Raschke GF, Rieger UM, Bader RD, Schaefer O, Guentsch A, Schultze-Mosgau S. Transconjunctival versus subciliary approach for orbital fracture repair-an anthropometric evaluation of 221 cases. Clin Oral Investig. 2013;17(3):933-42. DOI: 10.1007/s00784-012-0776-3.

14. Pedemonte C, Sáez F, González E, Vargas I. Acceso retrocaruncular para la reconstrucción de la pared medial de la órbita. Rev Esp Cir Oral y Maxilofac. 2016;38(4):206-12. DOI: 10.1016/j. maxilo.2015.10.001.

15. Bernardini FP, Nerad J, Fay A, Zambelli A, Cruz AA V. The Revised Direct Transconjunctival Approach to the Orbital Floor. Ophthalmic Plast Reconstr Surg. 2017;33(2):93-100. DOI: 10.1097/IOP.0000000000000659.

16. Yamashita M, Kishibe M, Shimada K. Incidence of lower eyelid complications after a transconjunctival approach: Influence of repeated incisions. J Craniofac Surg 2014;25(4): 1183-6. DOI: 10.1097/SCS.0000000000000836. 